\section{Enfermedad diverticular: mitos y realidades}

\author{
DANIELA FLUXÁ ${ }^{1, \mathrm{a}}$, RODRIGO QUERA ${ }^{1,2}$
}

\section{Diverticular disease: myths and realities}

Diverticulosis and diverticular disease of the colon are common conditions in Western countries. The incidence and prevalence of these diseases are increasing and becoming significant for health systems. A growing body of knowledge is shifting the paradigm of the pathogenesis and treatment of diverticular disease. Low-grade inflammation, altered intestinal microbiota, visceral hypersensitivity, and abnormal colonic motility have been identified as factors leading to diverticular disease. The risk of developing diverticulitis among individuals with diverticulosis is lower than 10 to $25 \%$. Studies indicate that diverticular disease may become a chronic disorder in some patients, not merely an acute illness. Contrary to the advice from international guidelines, studies have not shown that a high-fiber diet protects against diverticulosis. The evidence about the use of antibiotics in uncomplicated diverticulitis is sparse and of low quality. In relation to surgery, studies support a more conservative approach to prophylactic surgery in patients with recurrent disease or chronic symptoms. Finally, new pathophysiological knowledge suggests that other treatments may be useful (mesalamine, rifaximin and probiotics). However, more research is necessary to validate the safety, effectiveness and cost-effectiveness of these strategies.

(Rev Med Chile 2017; 145: 209-218)

Key words: Colonic Diseases; Diverticulosis; Diverticulitis, Colonic.

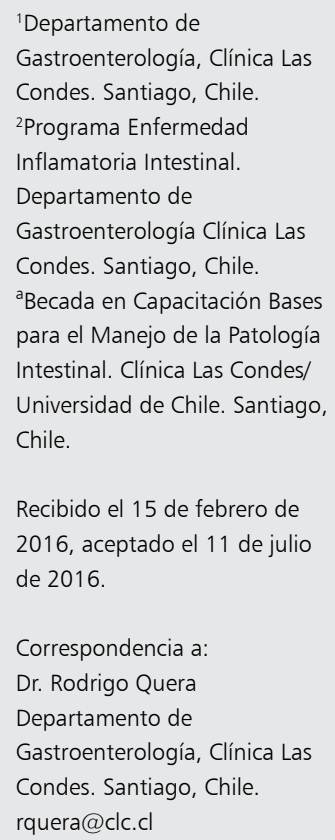

I a enfermedad diverticular (ED) es uno de los diagnósticos gastroenterológicos más frecuentes, presentando un aumento de 15\% en el número de hospitalizaciones ${ }^{1}$. Al hablar de ED se debe separar las anormalidades anatómicas del síndrome clínico. La diverticulosis describe el desarrollo de formaciones saculares de la mucosa y submucosa a través de áreas con capa muscular más delgada, donde los vasos sanguíneos penetran la pared intestinal ${ }^{2}$. Esta patología debe ser diferenciada de divertículos encontrados en otras áreas del tracto gastrointestinal, incluyendo intestino delgado y colon ascendente ${ }^{3}$. La ED describe cualquier cuadro que se origina como consecuencia detener divertículos (Figura 1).

El tratamiento y manejo actual de la ED se basa aún en teorías o estudios realizados décadas atrás. Estudios recientes han cuestionado muchos de estos dogmas al revelar antecedentes epidemio- lógicos, fisiopatológicos, clínicos y terapéuticos. El objetivo de esta revisión es entregar una visión actualizada, que permita un mejor enfrentamiento de esta patología por parte de médicos generales y especialistas.

\section{Epidemiología}

La diverticulosis y la ED son frecuentes en países industrializados. La incidencia de la diverticulosis aumenta desde $<16 \%$ en pacientes menores de 40 años a $>63 \%$ en mayores de 70 años $^{4}$. Su ubicación más frecuente es a nivel de sigmoides, sin embargo, en países de Asia se localizan principalmente en colon ascendente $e^{5}$.

La historia natural de la diverticulosis no está aclarada. Mientras la mayoría de los pacientes tienen diverticulosis, se pensaba tradicionalmente 


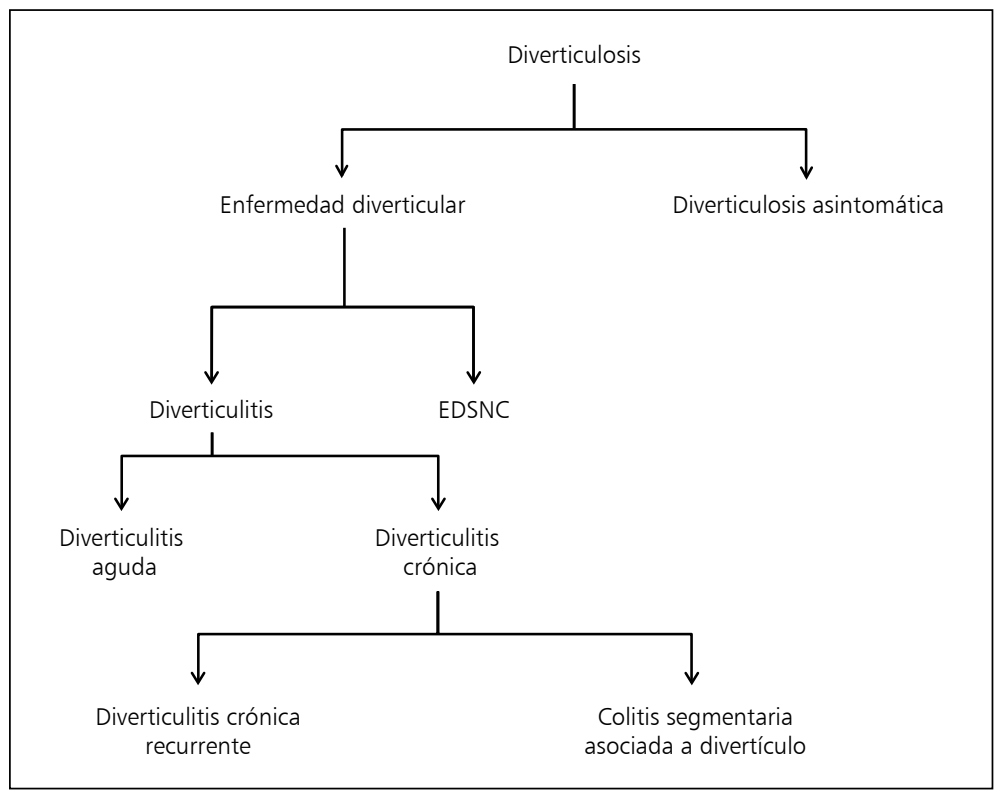

Figura 1. Términos relacionados con la diverticulosis y la enfermedad diverticular. Enfermedad diverticular sintomática no complicada (EDSNC). que $15-25 \%$ de ellos desarrollaría una diverticulitis aguda (DA) durante su vida ${ }^{6,7}$. Sin embargo, estos datos están basados en estudios de pesquisa de cáncer colorectal (CCR) por colonoscopia, lo que subvalora el número real de pacientes con diverticulosis. Estudios recientes han planteado que la incidencia de DA podría ser $<5 \%{ }^{8}$. Es importante mencionar que pacientes jóvenes presentarían una mayor incidencia de diverticulitis por año de vida en comparación a población de mayor edad, cuestionando la percepción tradicional acerca de la frecuencia de progresión de diverticulosis a $\mathrm{DA}^{9}$.

\section{Patogenia}

La patogenia de la diverticulosis impresiona ser multifactorial. Factores como baja ingesta de fibras, dismotilidad intestinal, microbiota intestinal(MI) y alteraciones del tejido conectivo han sido propuestos en su desarrollo y evolución (Figura 2). La edad pareciera ser el factor de riesgo más importante en la diverticulosis ${ }^{3}$. Sin embargo, cambios en el tejido conectivo, como un aumento en el reticulado de las fibras de colágeno o la alteración en la expresión de metaloproteinasas más que la edad por sí misma, explicarían este riesgo ${ }^{10,11}$.

La DA era tradicionalmente explicada por fecalitos que obstruían el divertículo causando un barotrauma, abrasión de la mucosa, inflamación y sobrecrecimiento bacteriano. Además, alimentos pobremente digeridos podían ubicarse dentro del divertículo causando trauma, isquemia, necrosis focal y microperforación ${ }^{12}$. Estudios recientes enfatizan el rol de mecanismos anatómicos, inflamación localizada, cambios de la microbiota $\mathrm{y}$ alteraciones de la motilidad como potenciales factores etiológicos (Figura 2). El tabaco puede aumentar en $23 \%$ el riesgo de $\mathrm{ED}^{13}$. Aspirina, antiinflamatorios no esteroidales (AINE) y opiodes aumentarían el riesgo de perforación y absceso en pacientes con $\mathrm{DA}^{14}$. Por otra parte, bloqueadores de canales de calcio y estatinas tendrían un efecto protector $^{15,16}$.

\section{Genética}

Aunque la patogenia de la ED ha sido enfocada a factores ambientales (principalmente dieta), estudios han sugerido que factores genéticos podrían contribuir al desarrollo y evolución de la diverticulosis. Un estudio demostró que el riesgo de ED era de 2,92 para los hermanos, comparado con la población general. Gemelos homocigotos presentarían un riesgo de 14,5 comparado con 5 en dicigotos ${ }^{17}$. Otros han señalado el rol del gen TNFSF15 en el riesgo de cirugía en pacientes con $\mathrm{DA}^{18}$.

Enfermedades genéticas relacionadas con alteraciones del tejido conectivo, como el síndro- 


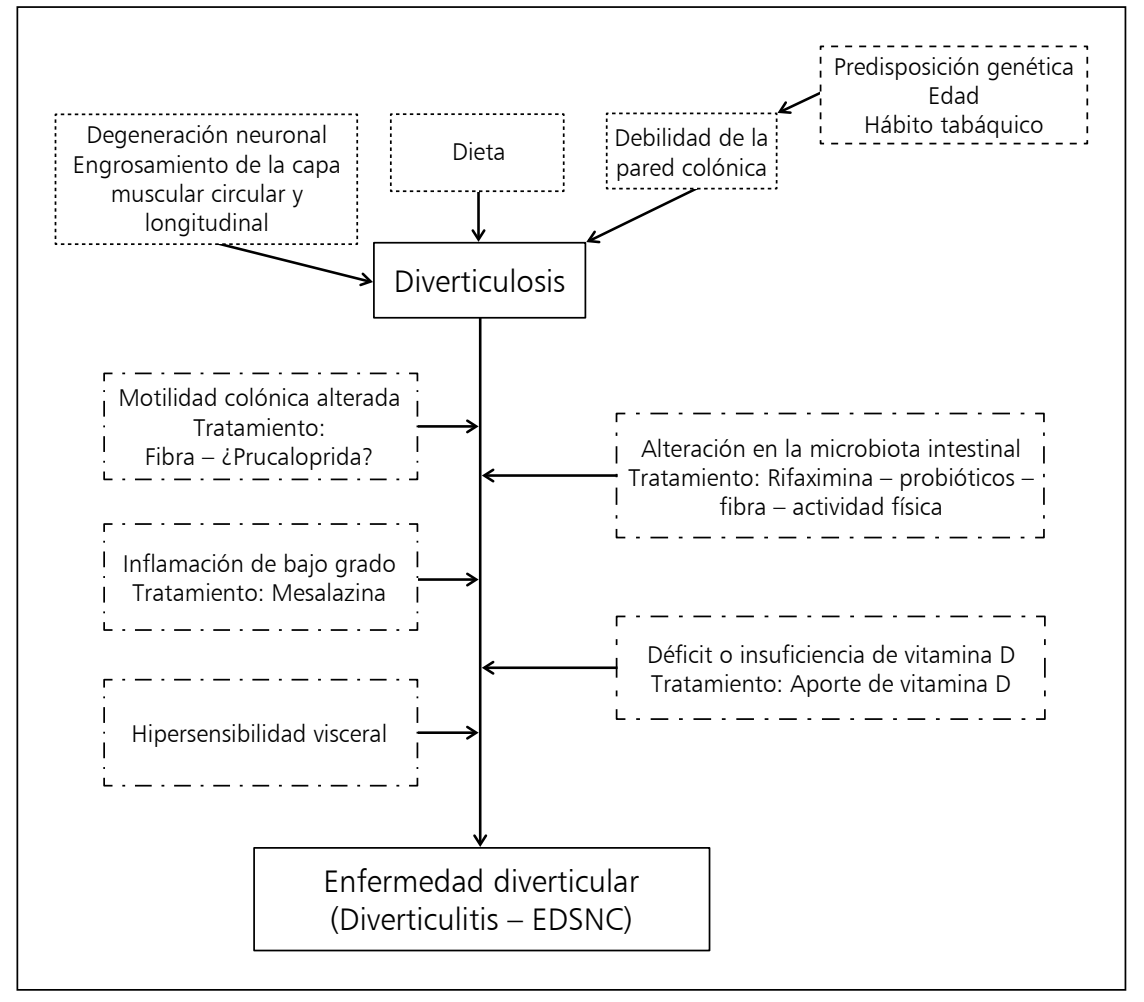

Figura 2. Patogenia y estrategias terapéuticas de la Diverticulosis y la Enfermedad Diverticular. Enfermedad Diverticular sintomática no complicada (EDSNC). me de Williams Beuren y Ehlers Danlos, estarían asociadas a diverticulosis, dado cambios a nivel de la elastina ${ }^{19}$ y disminución de la expresión de colágeno- $3^{20}$, respectivamente.

\section{Hábito intestinal}

Existe evidencia que contradice la hipótesis que la constipación esté asociada al desarrollo de divertículos. Un estudio encontró que la constipación se asoció a una disminución en el riesgo de diverticulosis, principalmente a nivel de colon descendente-sigmoides. Por otra parte, la diarrea no tuvo relación con esta patología ${ }^{21}$. Otros han confirmado la asociación entre el número de deposiciones y el riesgo de diverticulosis ${ }^{22}$. Pacientes con menos deposiciones ( $<7 /$ semana) presentaron menos divertículos comparado con pacientes con un número mayor ( $>7 /$ semana).

\section{Dieta}

Tradicionalmente, la dieta ha sido considerada un factor importante en el desarrollo de diverticulosis y su progresión a ED. Sin embargo, es difícil definir si los cambios en esta son causa o efecto de la ED. Sin duda, la evaluación de una población sana para la pesquisa del CCR con colonoscopía ha permitido evaluar la población con diverticulosis. Estudios iniciales sugirieron el rol de una dieta baja en fibras como un factor causal ${ }^{23}$. Estudios actuales sugieren que una dieta rica en fibras podría incluso aumentar el riesgo de $\mathrm{ED}^{4}$. Sin embargo, otros han confirmado una disminución al ingerir una dieta rica en fibras, especialmente cereales y frutas ${ }^{24,25}$. Por otra parte, una elevada ingesta de carnes rojas podría ser el factor más importante en el desarrollo de $\mathrm{ED}^{25}$. Es importante mencionar que estudios han descartado que la ingesta de nueces, semillas y maíz sean causal de una $\mathrm{DA}^{26}$.

Finalmente, aunque las recomendaciones varían con respecto a las modificaciones de la dieta en pacientes con DA, no existe evidencia que apoye una restricción de esta en DA no complicada ${ }^{27}$. Un estudio demostró que los médicos indican muchas veces restricciones de la dieta independiente de la severidad de la DA, lo que conlleva una prolongación innecesaria de hospitalización ${ }^{28}$. Sin duda, se requieren estudios para definir cuál es el rol de la fibra y dieta en el desarrollo y manejo de la ED. 


\section{Obesidad}

Estudios han señalado que pacientes con IMC $\geq 30 \mathrm{Kg} / \mathrm{m}^{2}$ presentan un mayor riesgo de DA y hemorragia diverticular al compararlos con sujetos con IMC $<21 \mathrm{~kg} / \mathrm{m}^{2(29)}$. Es más, pacientes con IMC $\geq 30 \mathrm{Kg} / \mathrm{m}^{2}$ presentan RR de 4,4 de ser hospitalizados por ED. Probablemente, la grasa visceral aumenta este riesgo, dado su rol en las complicaciones metabólicas e intestinales. Un estudio demostró que aquellos que tenían un área de grasa visceral $\geq 100 \mathrm{~cm}^{2}$ presentarían mayor riesgo de $\mathrm{DA}^{30}$.

El aumento de la grasa a nivel del mesenterio estaría asociado a una activación de macrófagos dentro del tejido adiposo, que llevaría a una acumulación de citoquinas proinflamatorias ${ }^{31}$. Los efectos de la grasa visceral sobre el deterioro de la función de barrera de la mucosa y la presencia de translocación bacteriana no pueden ser descartados en el desarrollo de la ED.

\section{Microbiota}

Cambios de dieta desde una basada en carbohidratos a una con mayor contenido en carnes conlleva alteraciones de la MI y su metabolismo ${ }^{32}$. La estasia fecal puede producir disbiosis y con ello inflamación crónica. Un estudio que incluyó pacientes con DA no complicada mostró que el $60 \%$ presentaba sobrecrecimiento bacteriano intestinal $^{33}$. Recientemente, un estudio comparó microbiota fecal de pacientes con diverticulitis y sujetos controles. Los resultados demostraron que la relación Firmicutes/Bacteroidetes y la carga de proteobacterias eran comparables. Sin embargo, una mayor diversidad de proteobacterias y de todos los phyla fue encontrada en pacientes con diverticulitis ${ }^{34}$. Estudios deberán definir el papel de la microbiota en la ED. La actividad física ha demostrado ser una medida efectiva en el manejo de la ED, probablemente por su efecto sobre la microbiota $^{35,36}$.

\section{Cuadro clínico}

\section{Enfermedad diverticular sintomática no complicada (EDSNC)}

La ED ha sido históricamente conceptualizada como asintomática o como una DA. Aunque el rol de la inflamación en la DA ha sido aceptado, estudios recientes proponen que algunos pacientes pueden evolucionar con síntomas debido a una inflamación crónica de bajo grado, condición que ha sido definida como EDSNC. Su prevalencia es incierta dado la sobreposición con el síndrome de intestino irritable (SII) (Figura 3). Un estudio menciona que en pacientes sin antecedentes de patología intestinal funcional, el desarrollo de SII era 4,7 veces más probable tras un episodio de $\mathrm{DA}^{37}$. Pacientes con EDSNC presentarían mayor percepción de dolor a la distensión, no solo del sigmoides, sino también del recto, confirmando la hipersensibilidad visceral ${ }^{9}$. Aumento de neuropéptidos y alteraciones en la inervación entérica han sido sugeridos como mecanismos relacionados con la hipersensibilidad ${ }^{38}$. Factores psicológicos también han sido asociados con dolor recurrente en pacientes con diverticulosis ${ }^{39}$. Trastornos de motilidad intestinal también han sido descritos en EDSNC, especialmente en segmentos que presentan divertículos ${ }^{40}$. Pacientes con diverticulosis presentarían una disminución de las células de Cajal al compararlo con sujetos controles ${ }^{41}$. Estas

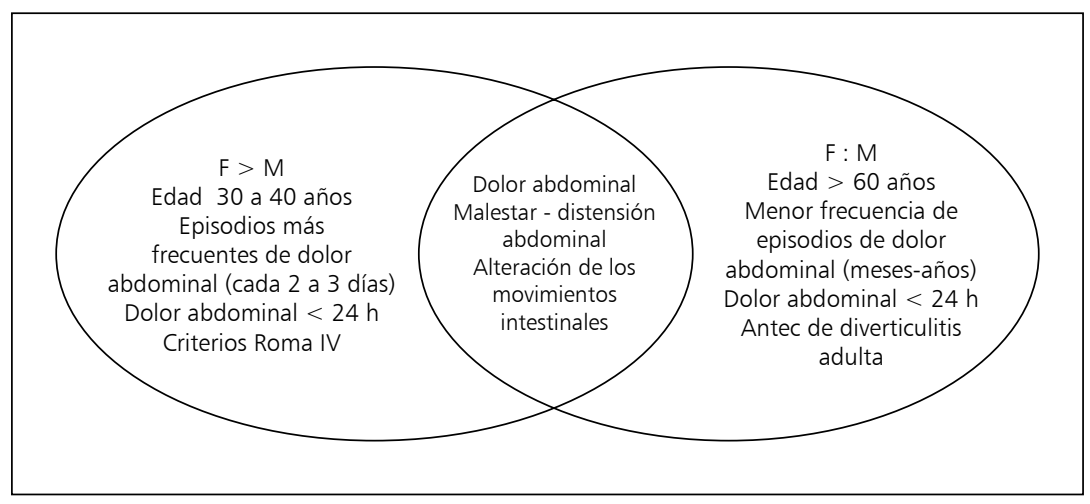

Figura 3. Sobreposición entre enfermedad diverticular sintomática no complicada (EDSNC) y síndrome intestino irritable (SII). 
asociaciones han permitido sugerir el término de SII post-diverticulitis, de manera similar al SII post-infeccioso ${ }^{42}$.

Dado que la EDSNC ocurre con mayor frecuencia después de los 60 años y el SII presenta un cenit entre los 30 y 40 años, el mayor desafío es diferenciar entre estos dos cuadros cuando se presentan en pacientes sobre 60 años (Figura 3). Estudios han señalado que la ubicación del dolor abdominal permitiría diferenciar estas dos pato$\operatorname{logías}^{43}$. La calprotectina fecal (CF) sería mayor en pacientes con EDSNC versus pacientes con diverticulosis, SII o sujetos sanos ${ }^{44}$.

\section{Diverticulitis aguda}

Tradicionalmente, la severidad de la DA era definida según la clasificación de Hinchey, que se basa en aspectos radiológicos e intraoperato$\operatorname{rios}^{45,46}$. Recientemente, estudios han sugerido que este cuadro podría ser dividido según las características clínicas y radiológicas (Tabla $1)^{47}$. Esta clasificación permite definir severidad y manejo de un evento de DA sin abarcar otros parámetros como recurrencia, refractariedad o colitis segmentaria (CS).

Los pacientes con respuesta clínica favorable posterior a un episodio de DA no complicada, pero que mantienen valores elevados de CF, tendrían mayor probabilidad de recurrencia ${ }^{48}$.Colonoscopías realizadas posterior a una DA no complicada muestran la presencia de inflamación endoscópica e histológica en 27 y $37 \%$, siendo considerados como predictores de recurrencia ${ }^{49}$.

\section{Colitis segmentaria}

Un pequeño subgrupo de pacientes puede desarrollar una CS asociada a diverticulosis ${ }^{50}$ (Figura
1). Su etiología no está aclarada, sin embargo, una disregulación inmunológica en respuesta a la MI ha sido sugerida ${ }^{51}$. Se estima que $1.3 \%$ de los pacientes con diverticulosis en la colonoscopía presentan hallazgos que sugieren esta patología ${ }^{52}$. Las lesiones son similares a las encontradas en enfermedad inflamatoria intestinal (EII), destacando la presencia de eritema, friabilidad, equimosis, erosiones o úlceras. A diferencia de la EII, el compromiso de mucosa rectal no está presente. Es importante considerar que las alteraciones histológicas también pueden recordar a esas presentes en EII, pudiéndose observar criptitis, abscesos crípticos, distorsión de la arquitectura glandular e incluso granulomas ${ }^{50}$. Aproximadamente $10 \%$ de los pacientes con CS pueden evolucionar hacia una EII, demostrando el rol que puede tener la inflamación en el desarrollo y evolución de la $\mathrm{ED}^{53}$.

\section{Tratamiento}

La ED confiere importantes costos en salud. Estudios realizados en Estados Unidos de Norteamérica (EEUU) han demostrado que el número de admisiones por ED alcanza 300.000 hospitalizaciones/año, con un costo anual de 2,4 billones de dólares ${ }^{54}$. Aunque las hospitalizaciones debido a ED han aumentado, las secundarias a perforaciones se han mantenido estables ${ }^{55}$. Esto indica que el mayor número de ingresos se debería a ED no complicada, por lo que su manejo debe reevaluarse. En la medida que la ecotomografía abdominal y tomografía computada (TC) de abdomen-pelvis resultan más accesibles y el conocimiento que la DA no complicada es más autolimitada, la proporción de pacientes de este

Tabla 1. Comparación de la Clasificación de Hinchey y la Clasificación de la Diverticulitis Aguda (DA)

$\begin{array}{ll}\begin{array}{ll}\text { Ia Inflamación o flegmón pericólica } \\ \text { confinado }\end{array} & \begin{array}{l}\text { DA no complicada } \\ \text { Presentación: fiebre, dolor abdominal y cambio en hábito intestinal } \\ \text { Imágenes: inflamación localizada o pequeño absceso en la pared intestinal } \\ \text { Tratamiento: puede ser manejado en forma ambulatoria; dieta liviana, uso de anti- } \\ \text { bióticos con discresión }\end{array} \\ \begin{array}{ll}\text { Ib Absceso localizado (pericolónico) } \\ \text { II Absceso pélvico }\end{array} & \begin{array}{l}\text { DA complicada } \\ \text { III Peritonitis purulenta }\end{array} \\ \text { IV Peritonitis fecaloídea } & \begin{array}{l}\text { Imágenes: inflamación pélvica o absceso distal, fístula, obstrucción intestinal } \\ \text { Tratamiento: paciente hospitalizado, antibióticos, eventual drenaje por radiología } \\ \text { intervencional o cirugía }\end{array}\end{array}$


grupo tratados de manera ambulatoria debería aumentar, disminuyendo las hospitalizaciones y costo económico asociado. Esta estrategia es efectiva en más de $90 \%$ de los pacientes, sin aumentar el riesgo del pequeño subgrupo que finalmente necesitará hospitalización ${ }^{56}$.

\section{Vitamina-D}

Estudios sugieren el rol de la vitamina-D en mantener la homeostasis colónica a través de la modulación de la inflamación, manteniendo la integridad epitelial y regulando la proliferación intestinal. Estudios han demostrado que niveles de vitamina-D $>25 \mathrm{ng} / \mathrm{mL}$ se asocian a un menor riesgo de hospitalización por $\mathrm{DA}^{57}$.

\section{Fármacos}

La inflamación crónica de bajo grado ha sido considerada un nuevo objetivo en el tratamiento de la ED. Su conocimiento permitirá una mejor estrategia terapéutica, mejorando la calidad de vida de estos pacientes. En este sentido, mesalazina, probióticos y antibióticos han sido evaluados en el tratamiento de la ED (Figura 2). Sin embargo, los resultados provienen de estudios heterogéneos y calidad subóptima.

Un estudio evaluó la eficacia y seguridad de mesalazina en la $\mathrm{EDSNC}^{58}$, aunque mostró ser bien tolerada, no fue superior a placebo en el tratamiento del dolor abdominal. Otro estudio evaluó el efecto de mesalazina en la prevención de DA. El grupo que utilizó mesalazina por 10 días cada mes, presentó a los 24 meses una recurrencia de 13\% vs $28 \%$ en la población control. Pacientes tratados con mesalazina presentaron, además, una mejoría de su calidad de vida y un menor uso de fármacos adicionales para el manejo de sus síntomas ${ }^{59}$. Mesalazina en dosis diaria (1,6 g/día) parece ser más efectiva en evitar recurrencia y complicaciones post-diverticulitis al compararla con una estrategia de mesalazina intermitente $(1,6 \mathrm{~g}$ /día por 10 días cada mes $)^{60}$. Otros han demostrado que pacientes que responden a mesalazina presentarían una disminución de la $\mathrm{CF}^{61}$. Sin embargo, no todos los estudios han entregado resultados optimistas sobre el efecto de mesalazina en el tratamiento de la ED. Recientemente, un estudio que evaluó tres dosis de mesalazina $(1,2 ; 2,4$ y 4,8 g día) no demostró efectividad sobre frecuencia de recurrencia comparada con placebo $^{62}$. Además, mesalazina ha demostrado tener un rol en $\mathrm{CS}^{63}$.
La asociación mesalazina-probióticos también ha mostrado resultados contradictorios. Stollman y cols. han evaluado la asociación de mesalazina (2,4 g/día) y probióticos (Bifidobacterium infantis) sin demostrar mayor efectividad. Mesalazina como monoterapia tampoco logró diferencias significativas versus placebo, al evaluar la evolución de los síntomas digestivos ${ }^{64}$. Otros demostraron que la recurrencia con la asociación mesalazina-probióticos fue de $0 \%$ vs $13,7 \%$ en el grupo tratado con mesalazina, $14,5 \%$ en el tratado con Lactobacillus y $46 \%$ en el grupo placebo ${ }^{65}$.

Dado que estudios han sugerido que la MI podría contribuir en la patogenia de la EDSNC, el uso de rifaximina ha sido evaluado en este escenario. Un metaanálisis demostró que rifaximina es efectivo en disminuir los síntomas con un NNT: ${ }^{66}$. Por otra parte, rifaximina $(800 \mathrm{mg} /$ día por 10 días cada mes) podría ser efectivo en prevenir diverticulitis ${ }^{67}$. Sin embargo, otros demostraron que la terapia combinada (mesalazina-rifaximina) es más efectiva en la prevención de recurrencia al compararla con rifaximina sola ( $3 \%$ vs $18 \%, \mathrm{p}<0,01)^{68}$.

A pesar de la falta de estudios que apoyen su uso, los antibióticos siguen formando parte del tratamiento de la DA. El fundamento de esta estrategia ha sido que cualquier inflamación resulta en microperforación del divertículo, teniendo como consecuencia contaminación del peritoneo. Sin embargo, estudios recientes plantean la necesidad de reevaluar esta indicación en casos no complica$\operatorname{dos}^{69,70}$. Chabok y cols. demostraron que el uso de antibióticos no afectaría la duración de la hospitalización o riesgo de recurrencia ${ }^{69}$. Considerando el aumento en la resistencia a antibióticos y riesgo asociado de eventos adversos, creemos prudente reevaluar esta estrategia en pacientes con DA no complicada.

\section{Cirugía}

Históricamente, la evaluación de cirugía profiláctica era sugerida después del segundo episodio de DA. Actualmente esta indicación ha sido replanteada, señalándose que ésta debe ser discutida caso a caso considerando la severidad de los episodios, factores de riesgo del paciente, preferencia y calidad de vida ${ }^{71}$. Estudios han demostrado que la calidad de vida después de una cirugía electiva no difiere al compararla con el grupo tratado con cirugía de urgencia o terapia farmacológica ${ }^{72}$. Sin embargo, un metaanálisis señala que la calidad de 
vida en pacientes con diverticulitis no complicada es mejor post-cirugía laparoscópica versus tratamiento conservador ${ }^{73}$. Pese a esta controversia, es importante considerar que $22 \%$ de los pacientes puede persistir con síntomas digestivos post-cirugía, $<5 \%$ de las recurrencias serán complicadas después de un episodio de DA no complicada y, finalmente, el número de episodios de recurrencia no tiene relación con el riesgo de complicaciones ${ }^{74}$. Considerando estos argumentos, creemos necesario mantener una conducta conservadora en la indicación quirúrgica en pacientes con episodios de recurrencia o síntomas crónicos.

\section{Colonoscopía post-diverticulitis}

Históricamente, las guías sugerían la necesidad de realizar una colonoscopía después de la resolución del episodio de DA (6-8 semanas) con el fin de descartar la presencia de un cáncer. Sin embargo, esta indicación debe ser reevaluada ${ }^{75,76}$. Brar y cols. evaluaron el rendimiento de la colonoscopía después de un año post-DA. CRC y neoplasia clínicamente significativa fueron encontradas en 1,6\% y $9,2 \%$, respectivamente. $\mathrm{Al}$ momento de evaluar los resultados, el subgrupo con diverticulitis complicada presentó mayor incidencia de adenoma avanzado ( $18 \%$ vs $5 \%$ ) y cáncer invasor $(5,4 \%$ vs $0 \%)$ comparado con pacientes con diverticulitis no complicada. La edad del paciente y presencia de abscesos deben ser considerados como los factores de riesgo más importantes al momento de definir quienes requieren este procedimiento ${ }^{75}$.

En pacientes que no evolucionan en forma favorable después de 7-10 días de tratamiento, una colonoscopía precoz permitirá descartar otra causa que explique la persistencia de síntomas. En este grupo, hasta $17 \%$ puede presentar otra patología significativa ${ }^{77}$. Una colonoscopía precoz (3-11 días después del ingreso) puede ser tan efectiva o segura como aquella realizada de manera tardía (6-12 semanas después del ingreso) sin presentar mayor riesgo de complicaciones ${ }^{78}$.

Finalmente, una selección de pacientes de acuerdo al compromiso colónico permitirá definir con mayor seguridad la mejor opción terapéutica. La clasificación endoscópica para ED (DICA) ha sido recientemente desarrollada y validada para lograr este objetivo (Tabla 2$)^{79}$. Es más, en pacientes DICA-1, prucaloprida podría tener un rol en el control de síntomas ${ }^{80}$.
Tabla 2. Clasificación de la Evaluación de la Complicación e Inflamación Diverticular (DICA)

\begin{tabular}{|lc|}
\hline Items & Puntos \\
\hline Extensión de la diverticulosis & \\
Colon descendente & 2 \\
Colon ascendente & 1 \\
Número de divertículos (en cada distrito) & \\
Grado I: hasta 15 & 0 \\
Grado II: sobre 15 & 1 \\
Presencia de signos inflamatorias: & \\
Edema/Hiperemia & 1 \\
Erosiones & 2 \\
Colitis segmentaria asociado a divertículos & 3 \\
Presencia de complicaciones & \\
Rigidez del colon & 4 \\
Estenosis & 4 \\
Pus & 4 \\
Sangrado & 4 \\
\hline
\end{tabular}

DICA 1: de 1 a 3; DICA 2: de 4 a 7; DICA 3: > 7.

\section{Conclusión}

La patogenia de la ED ha sido recientemente cuestionada. La causa de la transición entre diverticulosis y ED es más compleja de los que se pensaba y probablemente multifactorial. El rol de la dieta y la motilidad colónica están siendo replanteados. El uso de antibióticos en el tratamiento de la DA no complicada debe ser reevaluado, individualizado y probablemente reservado para pacientes con infección severa, múltiples comorbilidades, inmunosuprimidos o con mal acceso a salud. Nuevas estrategias se han planteado en el manejo de la prevención de la recurrencia, sin embargo, estudios no han podido confirmar su eficacia. A la luz de los resultados, la indicación generalizada de cirugía profiláctica en caso de dos o más episodios de diverticulitis no complicada debe ser reconsiderada. En suma, en casos de DA recurrente el uso de rifaximina o rifaximina más mesalazina parecería ser una opción razonable previo a la cirugía profiláctica.

\section{Referencias}

1. Everhart JE, Ruhl CE. Burden of digestive diseases in the United States. Part II. Lower gastrointestinal diseases. Gastroenterology 2009; 136: 741-54.

2. Stollman N, Raskin JB. Diverticular disease of the colon. Lancet 2004; 363: 631-9. 
3. Commane DM, Arasaradnam RP, Mills S, Mathers JC, Bradburn M. Diet, ageing and genetic factors in the pathogenesis of diverticular disease. World J Gastroenterol 2009; 15: 2479-88.

4. Perry AF, Barrett PR, Park D, Rogers AJ, Galanko JA, Martin CK, et al. A high-fiber risk diet does not protect against asymptomatic diverticulosis. Gastroenterology 2012; 144: 266-72.

5. Yamada E, Inamori M, Uchida E, Tanida E, Izumi M, Takeshita K, et al. Association between the localization of diverticular disease and the irritable bowel syndrome: a multicenter study in Japan. Am J Gastroenterol 2014; 109: 1900-5.

6. Parks TG. Natural history of diverticular disease of the colon. Clin Gastroenterol 1975; 4: 53-69.

7. Stollman NH, Raskin JB. Diagnosis and management of diverticular disease of the colon in adults. Ad Hoc Practice Parameters Committee of the American College of Gastroenterology. Am J Gastroenterol 1999; 94: 311021.

8. Shahedi K, Fuller G, Bolus R, Cohen E, Vu M, Shah R, et al. Long-term risk of acute diverticulitis among patients with incidental diverticulosis found during colonoscopy. Clin. Gastroenterol Hepatol 2013; 11: 1609-13.

9. Clemens $\mathrm{CH}$, Samsom M, Roelofs J, van Berge Henegouwen GP, Smout AJ. Colorectal visceral perception in diverticular disease. Gut 2004; 53: 717-22.

10. Stumf M, Cao W, Klinge U, Klosterhalfen B, Kasper K, Schumpelick V. Increased distribution of collagen type III and reduced expression of matrix metalloproteinase 1 in patients with diverticular disease. Int J Colorectal Dis 2001; 16: 271-5.

11. Wess L, Eastwood MA, Wess TJ, Busuttil A and Miller A. Cross linking of collagen is increased in colonic diverticulosis. Gut 1995; 37: 91-4.

12. Stollman NH, Raskin JB. Diverticular disease of the colon. J Clin Gastroenterol 1999; 29: 241-52.

13. Hjern F, Wolk A, Hakansson N. Smoking and the risk of diverticular disease in women. Br J Surg 2001; 98: 907-1002.

14. Kvasnovsky CL, Papagrigoriadis S, Bjamason I. Increased diverticular complications with nonsteroidal anti-inflammatory drugs and other medications: a systematic review and meta-analysis. Colorectal Dis 2014; 16: O189-96.

15. Morris CR, Harvey IM, Stebbings WS, Speakman CT, Kennedy HJ, Hart AR. Do calcium channel blockers and antimuscarinics protect against perforated colonic diverticular disease?. A case control study. Gut 2003; 52: 1734-7.

16. Humes DJ, Fleming KM, Spiller RC, West J. Concurrent drug use and the risk of perforated colonic diverticular disease: a population-based case-control study. Gut 2011; 60: 219-24.

17. Strate LL, Erichsen R, Baron JA, Mortensen J, Pedersen $\mathrm{JK}$, Riis AH, et al. Heritability and familial aggregation of diverticular disease: a population-based study of twins and siblings. Gastroenterology 2013; 144: 736-42.

18. Connelly TM, Berg AS, Hegarty JP, Deiling S, Brinton D, Poritz LS, et al. The TNFSF15 gene single nucleotide polymorphism rs7848647 is associated with surgical diverticulitis. Ann Surg 2014; 259: 1132-7.

19. Santin BJ, Prasad V, Caniano DA, Colonic diverticulitis in adolescents: an index case and associated syndromes. Pediatr. Surg Int 2009; 25: 901-5.

20. Blaker H, Funke B, Hausser J, Hackert T, Schirmacher $\mathrm{P}$, Autschbach F. Pathology of the large intestine in patients with vascular type Ehlers Danlos syndrome. Wirchows Arch 2007; 450: 713-7.

21. Yamada E, Inamori M, Watanabe $S$, Sato T, Tagri M, Uchida E, et al. Constipation is not associated with colonic diverticula: a multicenter study in Japan. Neurogastroenterol Motil 2015; 27: 333-8.

22. Perry AF, Sandler RS, Ahnen DJ, Galanko HA, Holm AN, Saukat A, et al. Constipation and a low-fiber Diet are not associated with diverticulosis. Clin. Gastroenterol Hepatol 2013; 11: 1622-7.

23. Painter NS, Burkitt DP. Diverticular disease of the colon: a deficiency disease of Western civilization. Br Med J 1971; 2: 450-4.

24. Crowe FL, Balkwill A, Caims BJ, Appleby PN, Green J, Reeves GK, et al. Source of dietary fibre and diverticular disease incidence: a prospective study of UK women. Gut 2014; 63: 1450-6.

25. Crowe FL, Appleby PN, Allen NE, Key TJ. Diet and risk of diverticular disease in Oxford cohort of European Prospective Investigation into Cancer and Nutrition (EPIC): prospective study of British vegetarians and non-vegetarians. BMJ 2011; 343: d4131.

26. Strate LL, Liu YL, Syngal S, Aldoori WH, Giovannucci EL. Nut, corn, and popcorn consumption and the incidence of diverticular disease. JAMA 2008; 300: 907-14.

27. Vennix S, Morton DG, Hahnloser D, Lange JF, Bemelman WA. Systematic review of evidence and consensus on diverticulitis: an analysis of rational and international guidelines. Colorectal Dis 2014; 16: 866-78.

28. van de Wall BJ, Draaisma WA, van Iersel JJ, van del Kaaij R, Consten EC, Broeders IA. Dietary restrictions for acute diverticulitis: evidence-base or expert opinion? Int J Colorectal Dis 2013; 28: 1287-93.

29. Strate LL, Liu YL, Aldoori WH, Syngal S, Giovannucci EL. Obesity increases the risks of diverticulitis and diverticular bleeding. Gastroenterology 2009; 136: 115-22.

30. Yamada E, Ohkubo H, Higurashi T, Sakai E, Endo E, 
Takahashi H, et al. Visceral obesity asa risk factor for left-sided diverticulitis in Japan: a multicenter retrospective study. Gut Liv 2013; 7: 532-8.

31. Batra A, Siegmund B. The role of visceral fat. Dig Dis 2012; 30: 70-4.

32. Devkota S, Wang YW, Musch MW, Leone V, Fehlner-Peach H, Nadimpalli A, et al. Dietary-fat-induced taurocholic acid promotes pathobiont expansion and colitis inIL10(-/-) mice. Nature 2012; 487: 104-8.

33. Tursi A, Brandimarte G, Giorgetti GM, Elisei W. Assessment of small intestinal overgrowth in uncomplicated acute diverticulosis of the colon. World J Gastroenterol 2005; 11: 2773-6.

34. Daniels L, Budding AE, de Korte N, Eck A, Bogaards JA, Stockman HB, et al. Fecal microbiome analysis as a diagnostic test for diverticulitis. Eur J Clin Microbiol Infect Dis 2014; 33: 1927-36.

35. Hjern F, Wolk A, Hakansson N. Obesity physical inactivity and colonic diverticular disease requiring hospitalization in women: a prospective cohort study. Am J Gastroenterol 2012; 107: 296-302.

36. O'Sullivan O, Cronin O, Clarke SF, Murphy EF, Molloy MG, Shanahan F, et al. Exercise and the microbiota. Gut Microbes 2015; 6: 131-6.

37. Cohen F, Fuller G, Bolus R, Modi R, Vu M, Shahedi K, et al. Increased risk for irritable bowel syndrome after acute diverticulitis. Clin Gastroenterol Hepatol 2013; 11: 1614-9.

38. Simpson J, Sundler F, Humes DJ, Jenkins D, Wakelin $\mathrm{D}$, Scholefield JH, et al. Post inflammatory damage to the enteric nervous system in diverticular disease and its relationship to symptoms. Neurogastroenterol Motil 2009; 21: 847-e58.

39. Humes DJ, Simpson J, Neal KR, Scholefield JH, Spiller RC. Psychological and colonic factors in painful diverticulosis. Br J Surg 2008; 95: 195-8.

40. Bassotti G, Sietchiping-Nzepa F, De Roberto G, Chistolini F, Morelli A. Colonic regularcontractile frequency patterns in irritable bowel syndrome: the "spastic colon" revisited. Eur J Gastroenterol Hepatol 2004; 16: 613-7.

41. Bassotti G, BattagliaE, Bellone G, Dughera L, Fisogni S, Zambelli C, et al. Intersticial cells of Cajal, enteric nerves and glial.cells in colonic diverticular disease. J Clin Pathol 2005; 58: 973-7.

42. Cohen E, Fuller G, Bolus R, Modi R, Vu M, Shahedi K, et al. Increased risk for irritable bowel syndrome after acute diverticulitis. Clin Gastroenterol Hepatol 2013; 11: 1614-9.

43. Tursi A, Elisei W, Picchio M, Giorgetti GM, Brandimarte G. Moderate-to-severe and prolonged left lower abdominal pain is the best symptom characterizing symptomatic uncomplicated diverticular disease of the colon: a comparison with fecal calprotectin in clinical setting. J Clin Gastroenterol 2015; 49: 218-21.

44. Tursi A, Brandimarte G, Elisei W, Giorgetti GM, Inchingolo CD, Aiello F. Faecal calprotectin in colonic diverticular disease: a case-control study. Int J Colorectal Dis 2009; 24: 49-55.

45. Wasvary H, Turfah F, Kadro O, Beauregard W. Same hospitalization resection for acute diverticulitis. Am Surg 1999; 65: 632-5.

46. Hinchey EJ, Schaal PG, Richards GK. Treatment of perforated diverticular disease of the colon. Adv Surg 1978; 12: 85-109.

47. Klarenbeek Br, de Korte N, van der Peet DL, Cuesta MA. Review of current classifications for diverticular disease and a translation into clinical practice. Int J Colorectal Dis 2012; 27: 207-14.

48. Tursi A, Elisei W, Picchio M, Brandimarte G. Increased faecal calprotectin predicts recurrence of colonic diverticulitis. Int J Colorectal Dis 2014; 29: 931-5.

49. Tursi A, Elisei W, Giorgetti GM, Inchingolo CD, Nenna R, Picchio M, et al. Detection of endoscopic and histological inflammation after an attack of colonic diverticulitis is associated with higher diverticulitis recurrence. J Gastrointest Liver Dis 2013; 22: 13-9.

50. Lamps LW, Knapple WL. Diverticular disease-associated segmental colitis. Clin Gastroenterol Hepatol 2007; 5: 27-31.

51. Ierardi E, Hassan C, Zullo A, De Francesco V, Valle ND, Prencipe S, et al. Segmental colitis associated with diverticula: a rare clinical entity and new challenge for the gastroenterologist. Dig Liver Dis 2009; 41: 794-7.

52. Tursi A, Elisei W, Brandimarte G, Giorgetti GM, Lecca PG, Di Cesare L, et al. The endoscopic spectrum of segmental colitis associated with diverticulosis. Colorectal Dis 2010; 12: 464-70.

53. Mulhall A, Mahid SS, Petras RE, Galandiuk S. Diverticular disease associated with inflammatory bowel disease-like colitis: a systematic review. Dis Colon Rectum 2009; 52: 1072-9.

54. Strate LL, Modi R. Cohen E, Spiegel BM. Diverticular disease as a chronic illness: evolving epidemiologic and clinical insights. Am J Gastroenterol 2012; 107: 1486-93.

55. Ricciardi R, Baxter NN, Read TE, Marcello PW, Hall J, Roberts PL. Is the decline in the surgical treatment for diverticulitis associated with an increase in complicated diverticulitis? Dis Colon Rectum 2009; 52: 1558-63.

56. Alonso S, Pera M, Pares D, Pascual M, Gil MJ, Courtier R,et al. Outpatient treatment of patients with uncomplicated acute diverticulitis. Colorectal Dis 2010; 12: e278-282.

57. Maguire LH, Song M, Strate LE, Giovannucci EL, Chan AT. Higher serum levels of vitamin D are associated 
with a reduced risk of diverticulitis. Clin Gastroenterol Hepatol 2013; 11: 1631-5.

58. Kruis W, Meier E, Schumacher M, Mickisch O, Greinwald R, Mueller R, et al. Randomised clinical trial: mesalazina (Salofalk granulate) for uncomplicated diverticular disease of the colon-a placebo controlled study. Aliment Pharmacol Ther 2013; 37: 680-90.

59. Parente F, Bargiggia S, Prada A, Bortoli A, Giacosa A, Germanà $B$, et al. Intermittent treatment with mesalazine in the prevention of diverticulitis recurrence: a randomized multicentre pilot double-blind placebo-controlled study of 24-month duration. Int J Colorectal Dis 2013; 28: 1423-31.

60. Tursi A, Di Mario F, Brabdimarte G, Elisei W, Picchio $\mathrm{M}$, Loperfido $\mathrm{S}$, et al. Intermittent versus every day mesalazine therapy in preventing complications of diverticular disease: a long-term follow-up study. Eur Rev Med Pharmacol Sci 2013; 17: 3244-8.

61. Tursi A, Brandimarte G, Elisei W, Giorgetti GM, Inchingolo CD, Aiello F. Faecal calprotectin in colonic diverticular disease: a case-control study. Int J Colorectal Dis 2009; 24: 49-55.

62. Raskin JB, Kamm MA, Jamal MM, Márquez J, Melzer E, Schoen RE, et al. Mesalamine does not prevent recurrent diverticulitis in phase 3 controlled trials. Gastroenterology 2014; 147: 793-802.

63. Imperiali G, Meucci G, Alvisi C, Fasoli R, Ferrara A, Girelli CM, et al. Segmental colitis associated with diverticula: a prospective study. Gruppo Di Studio Per Le Malatti e Inflammatorie Intestinali (GSMII). Am J Gastroenterol 2000; 95: 1014-6.

64. Stollman N, Magowan S, Shanahan F, Quigley E, DIVA Investigator Group. A randomized controlled study of Mesalamine after acute diverticulitis: results of the DIVA trial. J Clin Gastroenterol 2013; 47: 621-9.

65. Tursi A, Brandimarte G, Elisei W, Picchio M, Forti G, Pianese G, et al. Randomised clinical trial: mesalazine and/or probiotics in maintaining remission of symptomatic uncomplicated diverticula disease $-\mathrm{a}$ double-blind, randomised, placebo-controlled study. Aliment Pharmacol Ther 2013; 38: 741-51.

66. Bianchi M, Festa V, Moretti A, Ciaco A, Mangone M, Tomatore $\mathrm{V}$, et al. Meta-analysis: long-therm therapy with rifaximin in the management of uncomplicated diverticular disease. Aliment Pharmacol Ther 2011; 33: 902-10.

67. Lanas A, Ponce J, Bignamini A, Mearin F. One year intermittent rifaximin plus fibre supplementation vs fibre supplementation alone to prevent diverticulitis recurrence: a proof-of-concept study. Dig Liver Dis
2013; 45: 104-9.

68. Tursi A, Brandimarte G, Daffina R. Long-therm treatment with mesalazine and rifaximin vs. rifaximin alone for patients with recurrent attacks of acute diverticulitis of colon. Dig Liver Dis 2002; 34: 510-5.

69. Chabok A, Pahlman L, Hjern F, Haajaniemi S, Smedh K, AVOD Study Group. Randomized clinical trial of antibiotics in acute uncomplicated diverticulitis. $\mathrm{Br} \mathrm{J}$ Surg 2012; 99: 532-9.

70. Isacson D, Thorisson A, Andreasson K, Nikberg M, Smedh K. Outpatient, non-antiniotic management in acute uncomplicated diverticulitis: a prospective study. Int. Colorectal Dis 2015; 30: 1229-34.

71. Rafferty J, Shellito P, Hyman NH, Buie WD. Standards Committee of American Society of Colon and Rectal Surgeons. Practice parameters for sigmoid diverticulitis. Dis Colon Rectum 2006; 49: 939-44.

72. Bargellini T, Martellucci J, Tonelli P, Valeri A. Longterm results of treatment of acute diverticulitis: still lessons to be learned? Updates Surg 2013; 65: 125-30.

73. Andeweg CS, Berg R, Staal JB, Ten Broek RP, van Goor H. Patient-reported outcomes after conservative or surgical management of recurrence and chronic complaints of diverticulitis: Systematic review and meta-analysis. Clin. Gastroenterol Hepatol 2016; 14: 183-90.

74. Regenbogen SE, Hardiman KM, Hendren S, Morris Am. Surgery for diverticulitis in the 21 st century: a systematic review. JAMA Surg 2014; 149: 292-303.

75. Brar MS, Roxin G, Yaffe PB, Stranger J, Mac Lean AR, Buie WD. Colonoscopy following nonoperative management of uncomplicated diverticulitis may not be warranted. Dis. Colon Rectum 2013; 56: 1259-64.

76. Ou G, Rosenfeld G, Brown J, Chan N, Hong T, Lim H, et al. Colonoscopy after CT-diagnosed acute diverticulits: Is it really necessary? Can J Surg 2015; 58: 226-31.

77. Lahat A, Yahai H, Sakhnini E, Menachem Y, Bar-Meir S. Role of colonoscopy in patients with persistent acute diverticulitis. World J Gastroenterol 2008;14: 2763-6.

78. Lahat A, Yanai H, Menachem Y, Avidam B, Bar-Meir S. The feasibility and risk of early colonoscopy in acutediverticulitis: a prospective controlled study. Endoscopy 2007; 39: 521-4.

79. Tursi A, Brandimarte G, Di Mario F, Andreoli A, Annunziata ML, Astergiano M, et al. Development and validation of an Endoscopic Classification of Diverticular Disease of the Colon: The DICA Classification. Dig Dis 2015; 33: 68-76.

80. Tursi A. Prucalopride for the Treatment of Symptomatic Uncomplicated Diverticular Disease of the Colon. J Clin Gastroenterol 2016 [Epub ahead of print]. 